\title{
L1 and L2 reading skills in Dutch adolescents with a familial risk of dyslexia
}

\author{
Ellie R. H. van Setten ${ }^{\text {Corresp., }}{ }^{1,2}{ }^{\text {， Wim Tops }}{ }^{1}$ ， Britt E. Hakvoort ${ }^{3}$ ， Aryan van der Leij ${ }^{3}$, Natasha M. Maurits $^{2,4}$, \\ Ben A. M. Maassen ${ }^{1,2}$ \\ 1 Center for Language and Cognition Groningen (CLCG), Faculty of Arts, University of Groningen, Groningen, the Netherlands \\ 2 Research School of Behavioural and Cognitive Neurosciences (BCN), University Medical Center Groningen, University of Groningen, Groningen, the \\ Netherlands \\ 3 Research Institute of Child Development and Education, University of Amsterdam, Amsterdam, the Netherlands \\ 4 Department of Neurology, University Medical Center Groningen, University of Groningen, Groningen, the Netherlands
}

Corresponding Author: Ellie R. H. van Setten

Email address: e.r.h.van.setten@rug.nl

Background.The present study investigated differences in reading and spelling outcomes in Dutch and English as a second language (ESL) in adolescents with a high familial risk of dyslexia, of whom some have developed dyslexia (HRDys) while others have not (HRnonDys), in comparison to a low familial risk control group without dyslexia (LRnonDys). This allowed us to investigate the persistence of dyslexia in the first language (L1) and the effect of dyslexia on the second language (L2), which has, in this case, a lower orthographic transparency. Furthermore the inclusion of the HRnonDys group allowed us to investigate the continuity of the familial risk of dyslexia, as previous studies observed that the HRnonDys group often scores in between the HRDys and LRnonDys group, and whether these readers without reading deficits in Dutch, have more reading difficulties in ESL.

Methods. The data of three groups of adolescents were analyzed; 27 LRnonDys, 25 HRdys 25 HRnonDys. The mean age was 14;1 years; months, and 37 were male. All were native speakers of Dutch, attended regular secondary education (grade 7-10), and were non-native speakers of English. Using MANOVA the groups were compared on Dutch and English word reading fluency (WRF), spelling and vocabulary, Dutch pseudoword and loanword reading fluency, phonological awareness (PA), rapid automatized naming (RAN), and verbal short term and working memory. A repeated measures ANOVA was used to compare English and Dutch WRF, spelling and vocabulary directly within the three groups.

Results. The analyses revealed that the HRDys group had a deficit in both reading and spelling in Dutch and ESL. They also performed poorer than the LRnonDys group on all other measures. Effect sizes were especially large for pseudoword reading and the reaction times during the PA task. The HRnonDys group scored generally poorer than the LRnonDys group but this difference was only significant for Dutch pseudoword reading, PA reaction times and verbal short term memory. In general the HRDys and HRnonDys group scored similar in Dutch and English, except for English WRF where the HRDys group scored slightly better than expected based on their Dutch WRF.

Discussion. There was a high persistence of dyslexia. Adolescents with dyslexia had large impairments in reading and spelling, and reading related measures, both in Dutch and ESL. Despite high interindividual differences, an overall three-step pattern was observed. Adolescents in the HRnonDys group scored in between the HRDys and LRnonDys group, supporting the polygenetic origin of dyslexia and the continuity of the familial risk of dyslexia. The lower orthographic transparency did not have a negative effect on L2 reading, spelling and vocabulary, both in the HRnonDys and HRDys group. The latter group 
performed slightly better than expected in L2, which may be a result of the massive exposure to English and high motivation to use English by adolescents. 
2

4

5

\title{
L1 and L2 Reading Skills in Dutch Adolescents with a Familial Risk of Dyslexia
}

\author{
Ellie R.H. van Setten ${ }^{(1,2)}$ \\ Wim Tops ${ }^{(1)}$ \\ Britt E. Hakvoort (3) \\ Aryan van der Leij (3) \\ Natasha M. Maurits $(2,4)$ \\ Ben A.M. Maassen ${ }^{(1,2)}$
}

Author Note

(1) Center for Language and Cognition Groningen (CLCG), Faculty of Arts, University of Groningen, Groningen, the Netherlands (2) Research School of Behavioural and Cognitive Neurosciences (BCN), University Medical Center Groningen, University of Groningen,

Groningen, the Netherlands (3) Research Institute of Child Development and Education, University of Amsterdam, Amsterdam, the Netherlands (4) Department of Neurology, University Medical Center Groningen, University of Groningen, Groningen, the Netherlands We thank the master students who helped with the data collection. We are also thankful for the participation of the children and the commitment of their parents to this study. Correspondence concerning this article should be addressed to Ellie van Setten, Center for Language and Cognition Groningen, Faculty of Arts, University of Groningen, Oude Kijk in 't Jatstraat 26, 9712 EK Groningen, the Netherlands. Contact: e.r.h.van.setten@rug.nl 
Background.The present study investigated differences in reading and spelling outcomes

in Dutch and English as a second language (ESL) in adolescents with a high familial risk of dyslexia, of whom some have developed dyslexia (HRDys) while others have not (HRnonDys), in comparison to a low familial risk control group without dyslexia (LRnonDys). This allowed us to investigate the persistence of dyslexia in the first language (L1) and the effect of dyslexia on the second language (L2), which has, in this case, a lower orthographic transparency.

Furthermore the inclusion of the HRnonDys group allowed us to investigate the continuity of the familial risk of dyslexia, as previous studies observed that the HRnonDys group often scores in between the HRDys and LRnonDys group, and whether these readers without reading deficits in Dutch, have more reading difficulties in ESL.

Methods. The data of three groups of adolescents were analyzed; 27 LRnonDys, 25 HRdys 25 HRnonDys. The mean age was 14;1 years; months, and 37 were male. All were native speakers of Dutch, attended regular secondary education (grade 7-10), and were non-native speakers of English. Using MANOVA the groups were compared on Dutch and English word reading fluency (WRF), spelling and vocabulary, Dutch pseudoword and loanword reading fluency, phonological awareness (PA), rapid automatized naming (RAN), and verbal short term and working memory. A repeated measures ANOVA was used to compare English and Dutch WRF, spelling and vocabulary directly within the three groups.

Results. The analyses revealed that the HRDys group had a deficit in both reading and spelling in Dutch and ESL. They also performed poorer than the LRnonDys group on all other measures. Effect sizes were especially large for pseudoword reading and the reaction times during the PA task. The HRnonDys group scored generally poorer than the LRnonDys group but 
48 this difference was only significant for Dutch pseudoword reading, PA reaction times and verbal

49 short term memory. In general the HRDys and HRnonDys group scored similar in Dutch and

50 English, except for English WRF where the HRDys group scored slightly better than expected

51 based on their Dutch WRF.

52

Discussion. There was a high persistence of dyslexia. Adolescents with dyslexia had

53 large impairments in reading and spelling, and reading related measures, both in Dutch and ESL.

54 Despite high inter-individual differences, an overall three-step pattern was observed. Adolescents

55 in the HRnonDys group scored in between the HRDys and LRnonDys group, supporting the

56 polygenetic origin of dyslexia and the continuity of the familial risk of dyslexia. The lower

57 orthographic transparency did not have a negative effect on L2 reading, spelling and vocabulary,

58 both in the HRnonDys and HRDys group. The latter group performed slightly better than

59 expected in L2, which may be a result of the massive exposure to English and high motivation to

60 use English by adolescents. 
61

62

63

\section{L1 and L2 Reading Skills in Dutch Adolescents with a Familial Risk of Dyslexia}

Dyslexia is a specific learning disorder characterized by problems with accurate and/or

fluent word recognition, poor decoding, and poor spelling abilities at the word level (American

Psychiatric Association, 2013, p. 67), but it can have consequences for higher level reading comprehension skills and writing skills as well (Tops, Callens, Cauwenberghe, Adriaens, \& Brysbaert, 2013). Dyslexia is not a discrete disorder, there is no natural cut-off point that separates the reading skills of people with and without dyslexia (Shaywitz, Escobar, Shaywitz, Fletcher, \& Makuch, 1992). Furthermore, dyslexia is a heterogeneous disorder, as research has found that there are multiple genes and cognitive deficits that may affect the reading skills of people with dyslexia (Carrion-Castillo et al., 2017; Carrion-Castillo, Franke, \& Fisher, 2013; Pennington, 2006; Pennington et al., 2012). While a phonological processing deficit, especially a deficit in phonological awareness (PA), is often seen as the core problem of dyslexia (e.g. Ramus et al., 2003; van der Leij \& Morfidi, 2006), it is neither necessary nor sufficient to explain all cases of dyslexia at the individual level (Pennington et al., 2012). Other cognitive deficits linked to reading problems include deficits in rapid automatized naming (RAN; e.g. Kirby, Georgiou, Martinussen, \& Parrila, 2010; Norton \& Wolf, 2012; Papadopoulos, Spanoudis, \& Georgiou, 2016), verbal working and short-term memory (VST/WM; e.g. de Jong, 1998; Pennington \& Lefly, 2001; Snowling, Gallagher, \& Frith, 2003) and orthographic processing (e.g. Georgiou, Papadopoulos, Zarouna, \& Parrila, 2012; Rothe, Cornell, Ise, \& Schulte-Körne, 2015). criteria used (Miles, 2004). However, as dyslexia has a partially genetic origin (e.g. CarrionCastillo et al., 2013) children with a parent with dyslexia have a much higher risk to develop dyslexia, on average 45 percent (Snowling \& Melby-Lervåg, 2016) . Because we can reliably 
84 predict, before formal reading instruction starts, that a large proportion of children with a (high)

85

86

87

88

89

familial risk of dyslexia (FR) will develop dyslexia, they constitute an interesting population for prospective longitudinal research. The participants of the present study have all participated in such a study in the Netherlands, the Dutch Dyslexia Program (DDP; See e.g. van Bergen, de Jong, Plakas, Maassen, \& van der Leij, 2012; van der Leij et al., 2013). In the DDP study, a group of children, of whom approximately two-third has a high FR and half of the children with a high FR has eventually developed dyslexia, has been followed from birth. The present study focused on a selection of these children at the age when they were advanced readers. More specifically, we investigated how (a family risk of) dyslexia influences the word level reading and spelling skills in early adolescence. As English is a mandatory subject in schools in the Netherlands, and is increasingly used in everyday life, we did not only examine reading and spelling in the participants' first language (L1) Dutch, but also reading and spelling in English, their second language (L2). To our knowledge this is the first large-scale study that involves adolescents with a high FR that has looked at L2 reading and spelling. Before we describe our research questions and hypotheses in more detail, we will first discuss general differences that have been found between (advanced) readers with and without (a familial risk of) dyslexia, the position of English in the Netherlands, and learning to read in English as a second language (ESL) with dyslexia.

\section{Readers with a Familial Risk of Dyslexia}

As dyslexia is not a discrete disorder, the degree at which parents are affected by dyslexia also varies. Hence, the familial risk for dyslexia is continuous as well. Some of the cognitive and reading impairments typically associated with dyslexia have also been found, usually in a milder form, in children with a high FR who do not have dyslexia (HRnonDys). In family risk studies 
107 they often score in between the group with a high FR with dyslexia (HRDys) and children with a 108 low FR without dyslexia (LRnonDys) in family risk studies (e.g. Elbro, Borstrøm, \& Petersen, 109 1998; Pennington \& Lefly, 2001; Snowling et al., 2003; van Bergen et al., 2012). This three-step 110 pattern is in line with a polygenetic inheritance and multifactorial origin of dyslexia, as the 111 HRnonDys group probably has inherited or has been exposed to some risk factors associated 112 with reading disability causing mild reading problems (see van Bergen, van der Leij, \& de Jong, 1132014 for a more in-depth discussion).

114 For advanced readers, results regarding this three-step pattern are somewhat mixed.

115 Snowling, Mutter and Carrol (2007) found significant differences in exception word reading, text 116 reading accuracy, and timed measures of nonsense passages, sight-word and decoding efficiency,

117 text reading rate, and spelling, between HRnonDys adolescents and a control group of 118 adolescents with a low familial risk without dyslexia (LRnonDys) at the age of 12-13. On the 119 other hand, Dandache, Wouters and Ghesquière (2014) found only a difference for Word 120 Reading Fluency (WRF) in Dutch readers in Grade 6, and Eklund, Torppa, Aro, Leppänen and 121 Lyytinen (2014) did not find significant difference between the HRnonDys group and the

122 LRnonDys group in Finnish readers in Grade 8. However, in all studies the scores of the 123 HRnonDys group were generally lower than the scores of the LRnonDys group, and higher than 124 the scores of the HRDys group. In all studies the HRDys group also scored significantly lower 125 than the LRnonDys control group. These effects were usually large, supporting the view that 126 dyslexia persists into adolescence, as has also been found by others (Ferrer et al., 2015; Shaywitz 127 et al., 1999).

\section{Position of English in the Netherlands}


Although Dutch is the dominant language in the Netherlands, English has a very

130

131

132

133

134

135

136

137

138

139

140

141

142

143

144

145

146

147

148

149

150

151

important position (See for a more elaborate discussion of the position of English in the

Netherlands also: Gerritsen, Van Meurs, Planken, \& Korzilius, 2016). According to McArthur

(1996) the high level of bilingualism between English and Dutch in the Netherlands justifies the

view that English is a strong second language, rather than a foreign language. In the Netherlands, children learn to read and write in their first language Dutch when they are 6 years old and enter grade 1. Explicit English L2 instruction is mandatory from grade 5 onwards and remains obligatory in almost all curricula until the end of secondary education and even in a wide range of fields of study in higher education. However, some schools already start English as early as Kindergarten, and the hours of English per week vary (Thijs, Trimbos, Tuin, Bodde, \& de Graaff, 2011). Dutch children come into contact with English almost daily via television and multimedia, like internet and games. According to Di Carlo (1994) contact with multimedia, where verbal messages are accompanied by visual images, has an impact on language learning. Moreover, it has been shown that watching subtitled programs and films in a foreign language causes (incidental) language learning, even in young children (d'Ydewalle \& Poel, 1999) because both languages are then simultaneously present.

\section{Reading in English (as a Second Language) with Dyslexia}

While English and Dutch both stem from Germanic origins, and share a relatively high syllabic complexity, they differ in the transparency of their letter-to-sound conversions. Dutch orthography has 40 phonemes that can be written in 76 ways, in contrast to English that has 44 phonemes that can be written in 561 ways (Dewey, 1971). Dutch orthography is therefore considered more transparent than English. In transparent orthographies, firm and accurate phonological representations are installed in the early stages of reading acquisition. Therefore 
152 phonological awareness and reading development are faster in transparent than in deeper

153 orthographies (Seymour, Aro, \& Erskine, 2003). This explains why a large proportion of Dutch

154 children can read accurately after one year of reading instruction, whereas English children need

155 on average four years to obtain the same reading level (Seymour et al., 2003).

156 According to the Linguistic Coding Differences Hypothesis (LCDH; Ganschow, Sparks,

157 Javorsky, Pohlman, \& Bishop-Marbury, 1991) individuals who encounter difficulties with L1

158 acquisition, will also have problems in L2 because there is a link in L1 and L2 learning,

159 particularly in learning to read. Ganshow et al. (1991) emphasized the importance of

160 orthographic, syntactic and semantic, and especially phonological skills to transition from L1 to

161 L2. Although the relation between phonological awareness and reading decreases with age,

162 phonological processing still plays a role in the reading skills of individuals with dyslexia at a

163 later age (Bekebrede, van der Leij, \& Share, 2009). For most typically developing children,

164 transition from L1 to L2 learning runs smoothly, whereas for children with dyslexia, L2 learning

165 is particularly challenging (Helland \& Kaasa, 2005). In transparent orthographies, children with

166 dyslexia encounter difficulties with reading speed. In deeper orthographies, such as English,

167 children with dyslexia experience difficulties with reading accuracy as well (Hagtvet \& Lyster,

168 2003).

169 In addition to phonological processing, orthographic processing is also necessary for

170 fluent reading. There is much evidence for differences in orthographic knowledge between

171 typical and dyslexic readers (Bekebrede et al., 2009). However, there is also evidence that a

172 subgroup of readers with dyslexia read better in English L2 than in their more transparent L1.

173 Miller-Guron and Lundberg (2000) found that a group of dyslexic adult students that had a self-

174 reported preference for English (L2) over Swedish (L1), indeed read better in English than in 
175 Swedish. Furthermore, the dyslexic students with a preference for English also performed better

176 on English spelling and free-writing tasks, compared to dyslexic students without a preference

177 for English. Miller-Guron and Lundberg (2000) have suggested that the dyslexic participants

178 with a preference for English could perhaps make use of frequently occurring spelling patterns or

179 orthographic structures that they learned from frequent exposure to English. According to the

180 authors, irregular grapheme-to-phoneme conversion rules in English dissuade readers from using

181 the decoding-route and favor the use of whole-word strategies. Additionally, van der Leij and

182 Morfidi (2006) found that a subgroup of Dutch (L1) poor readers in grade 7 and 8 were better at

183 English (L2) reading than at Dutch reading, and had a higher verbal competence in English than

184 they had in their mother tongue. This group was shown to have superior orthographic

185 competence, as measured with an orthographic choice task in both Dutch and English, compared

186 to a group of dyslexic readers who failed to show an advantage in L2 English.

\section{The Present Study}

The present study focused on Dutch and ESL literacy skills in Dutch adolescents with a

familial risk of dyslexia. As dyslexia is a problem at the word level we compared the three main groups, HRDys, HRnonDys and LRnonDys, on word level reading and spelling, in Dutch and

English, as well as on word-level reading related skills: RAN, PA, vocabulary and VST/WM.

Based on previous studies we expected large differences between the HRDys group and the expected that the HRnonDys group will perform in between the HRDys and LRnonDys group, but that these differences may not always be significant. As L1 reading skills are highly predictive for $\mathrm{L} 2$ reading skills according to the $\mathrm{LCDH}$ we expected that reading and spelling in

197 ESL is also highly problematic for the HRDys group. To investigate if the effects are similar 
198 across languages, we directly compared Dutch and English reading, spelling and vocabulary

199 skills between the groups. The lower orthographic transparency may make reading in English

200 more difficult, but a preference for reading in ESL has also been observed among some dyslexic

201 readers. Moreover, the inclusion of a HRnonDys group allowed us to investigate if this group,

202 which by definition has no reading problems in Dutch, does have reading difficulties in English

203 which is orthographically more complex than Dutch.

204

205

206

207

208

209

210

211

212

213

214

215

216

217

218

219

220

\section{Method}

Participants Eighty-one adolescents were included in the present study, 39 were male and 42 were female. They attended year 1 to 4 of Dutch secondary school, corresponding to grades 7-10 (mostly Grade 8), and the mean age was 14;1 years; months ( $S D=7$ months). They all participated in the Dutch Dyslexia Program (DDP) in Groningen or Amsterdam, the Netherlands. Seventy-eight percent of the children from Amsterdam and Groningen who participated in the previous DDP measurement in Grade 6, participated in the present study. All participants were native speakers of Dutch and spoke mainly Dutch in school, but a few participants in the Northern region also spoke Frisian at home. None of the participants had lived abroad in an English speaking country other than during a holiday.

Based on parental reading scores participants were divided into a high FR and low FR control group. Parents were tested with WRF and pseudoword reading fluency (PWRF) tasks, the same tasks that were used in this study and are described later. If the reading scores of at least one parent belonged to the lowest 20 percent on one test and to the lowest 40 percent on the other test, using the norms by Kuijpers et al. (2003), and if they reported a family history of dyslexia, participants were categorized in the high FR group. For the low FR control participants, reading scores of both parents did not meet these criteria, nor was there a self-reported family 
221 history of dyslexia. Dyslexia status was based on the participants' reading scores during previous

222 DDP measurements. The adolescents were categorized in the group with dyslexia if they met the

223 following criteria: participation in at least two out of three measurements in grades 2,3 , and 6 ,

224 two scores in the lowest 10\% range on either WRF or PWRF (again measured with the same

225 tests), and at least below average on the other test during that measurement. If they had

226 participated only twice and met the criteria once they were excluded because we aimed to

227 include only participants with persistent reading problems (one participant was excluded). By

228 combining the risk and dyslexia categorizations, four groups were created: 27 participants with a

229 low FR without dyslexia (LRnonDys), 25 participants with a high FR without dyslexia

230 (HRnonDys), 25 participants with a high FR with dyslexia (HRDys) and 4 participants with a

231 low FR with dyslexia (LRDys). While the latter group is too small for group comparisons, these

232 adolescents were included in the standardization of the test measures, which was based on the

233 whole low FR group, to have a more accurate estimation of the typical population. Participant

234 characteristics can be found in Table 1. Because of the longitudinal design groups could not be

235 matched on factors like age and gender. As age did not differ significantly between the three

236 main groups $(F(2,74)=2.085, p=.132)$, we did not further consider age in the analyses.

237 Exclusion criteria were an IQ below 80 as measured during previous assessments, severe

238 medical or psychiatric problems, or the attendance of a secondary school where the language of

239 instruction for other subjects than English was English. For the latter reason four participants

240 (two in each non-dyslexic group) were excluded from the study. Since the other exclusion

241 criteria were already applied during previous DDP assessments no participants were excluded for

242 these reasons. Participants with comorbid developmental disorders, such as Attention Deficit

243 Hyperactivity Disorder (ADHD), Attention Deficit Disorder (ADD) or Autism Spectrum 
244 Disorder (ASD) were included in the study as comorbidity is common between dyslexia and

245 other (neuro)-developmental problems (e.g. Germanò, Gagliano, \& Curatolo, 2010; Kaplan,

246 Wilson, Dewey, \& Crawford, 1998; Mayes \& Calhoun, 2006; Willcutt \& Pennington, 2000), and

247 excluding these participants would lead to an unrepresentative sample. Parents reported three

248 cases of comorbid disorders in the LRnonDys group (one with ASD and two with ADD), two in

249 the HRnonDys group (one with ASD and one with ADD), and four in the HRDys group (one

250 with ASD, two with ADHD, and one with ADD). Parents reported that one participant in the

251 LRDys group showed signs of both ADD and ASD, but it was unclear whether this participant

252 had an official diagnosis. From questionnaires conducted in Grade 6 we know that about 11

253 percent of the children in the LRnonDys group, 14 percent of the children in the HRnonDys

254 group, and 75 percent of the children in the HRDys group received extra help in school.

255 Materials

Reading Fluency. Four tests of reading fluency, measuring both reading accuracy and

257 speed, were included in the study: Dutch WRF ("Een minuut Test", one minute test, Brus \& Voeten, 1973), Dutch PWRF (“Klepel”, van den Bos, Lutje Spelberg, Scheepstra, \& de Vries, 1994), English WRF (Kleijnen, Steenbeek-Planting, \& Verhoeven, 2008), and loan word reading

fluency, using loanwords from English that are incorporated in the Dutch language (Schijf, 2009). The participants had to read aloud word lists, with words of increasing difficulty, as fast and accurately as possible. The score is the number of words read correctly within the allotted

263 time, which was one minute for all tests, except for PWRF where it was two minutes. The English WRF test had 108 words and the Loan word test 116 words; for each test the words were presented in four columns. For the Dutch WRF and PWRF the A-version was used, but the last 
266 column of the B-version was added, such that there were 145 words spread over 5 columns, to

267 ensure that participants would not finish reading all words within the allotted time. sentence was read, next the target word(s) were repeated. The participant had to write the target words on an answer sheet where the rest of the sentence was already printed. The test was executed at the participant's own pace and at request words were repeated more than once. For Dutch the "PI-signalerings dictee" (Geelhoed \& Reitsma, 2004) was used, which is a spelling screening instrument for the first grade of secondary school. It consists of 38 sentences and has 90 target words. For Dutch learners of English there was no standardized spelling test available. the first two grades of Dutch secondary school (grades 7 and 8). This dictation consists of 20 sentences with 20 target words, and can be found in Appendix A .

Vocabulary. Dutch and English vocabulary knowledge were tested with an oral vocabulary test. For Dutch this was the vocabulary task from the third edition of the Dutch Wechsler Intelligence Scale for Children (WISC-III-NL, Wechsler, 2005). During this task participants were asked to describe the meaning of words that increased in difficulty as the test progressed, like for example "paraplu" (umbrella) and "onafhankelijk" (independent). There are 35 items and 2 points can be gained for a fully correct answer and 1 point for a partially correct answer as determined by the test manual. After 4 incorrect answers the test is terminated. English vocabulary knowledge was tested with the fourth edition of the Peabody Picture Vocabulary Test (PPVT-IV, Dunn \& Dunn, 2007). During this test the participants heard a word and had to indicate which picture out of four pictures matches with the word. First a base set was established, a set of 12 items where all answers were correct or only one mistake was made. 
289 Thus, if more mistakes were made in the starting set, determined by the participant's age, a lower 290 set was chosen until the base set was reached. Next, the ceiling set was established, the set for 291 which eight or more mistakes were made, by moving up from the starting set. A total score is 292 obtained by subtracting the total number of errors from the item number of the last item of the 293 ceiling set. The maximum score for this test is 228.

294 Phonological Awareness. PA was measured with a Dutch spoonerism task (Depessemier $295 \&$ Andries, 2009). The test was computerized and the items were recorded such that all 296 participants heard the items exactly the same way. The participants heard two words through 297 headphones and were instructed to switch the first sounds of the words such that the first sound 298 of the second became the first sound of the first word, and vice versa. This resulted mostly in 299 pseudowords, but sometimes also in other real words. For example "nieuwe wagen" (new 300 wagon) became "wieuwe nagen". If a word started with a consonant cluster, both the answer where the whole cluster was reversed and the answers where a single phoneme was reversed 302 were considered correct. For example in the case of "grote boom" (big tree), both "brote goom" and "bote groom" were considered correct. There were six practice items and 20 test items. For the test items the participants were instructed to respond as fast and accurately as possible, but only if they knew the complete answer, such that the researcher could record both reaction times and accuracy. Reaction times were recorded manually when the participant started to utter the first word. The reaction times scores were corrected for extreme outliers and wrong registrations by removing reaction times shorter than $100 \mathrm{~ms}$ and longer than 3 standard deviations above the mean reaction time per participant.

311 \& Lutje Spelberg, 2007). The participant had to name 50 digits grouped in 5 rows as fast and 
312 accurately as possible. Taking into account accuracy and the time needed to name all items the

313 number of items that could be named correctly within one minute was calculated.

315 (Wechsler, 2005) was used to measure VST/WM. During the forward task, where mainly short

316 term memory is involved, the participants heard a series of digits that had to be repeated in the

317 same order. During the backward task the participant had to repeat the series of digits in a

318 reversed order. Here working memory is also involved as the information does not only have to

319 be stored but also has to be manipulated. The length of the series increases during both digit span

320 tasks. If the participant failed to repeat two series of the same length, the test is terminated. The

321 score is the number of series reported correctly. The maximum score is 16 in the forward task

322 and 14 in the backward task.

323 Procedure

Participants were seated in a quiet test environment. Tests were conducted individually.

As they participated in the DDP before, participants were already familiar with this testing situation. First, all Dutch tests were conducted, then the reading related subtests, and finally the English tests, to not confuse the participants with the different languages. There was a break in between the reading related tests and the English tests and participants were encouraged to take more breaks if deemed necessary. Informed consent was obtained from the parents, and adolescents gave their informed assent, as well. Travel costs were reimbursed and participants received a gift voucher to thank them for their participation. The study was approved by the ABR-nr: NL48140.042.14).

\section{Analyses}



use expectation maximization to impute missing data, a missing value analysis was conducted with Little's MCAR test in SPSS 23 (IBM Corp, Armonk, NY, USA). A Multivariate Analysis of Variance (MANOVA) was conducted between the LRnonDys, HRnonDys and HRDys groups

to investigate first if there was a group difference over all measures together, and next to investigate for which measures the groups differed from each other. Post-hoc pairwise comparisons with the Least Square Difference Method were used to investigate which groups differed from each other. Since we did overall tests first, and since we have separate hypotheses for each contrast, namely that we would see a three-step pattern for each of the included reading (related) test where the LRnonDys group would have the highest score and the HRDys group the lowest, we did not further correct for multiple comparisons as this would strongly reduce power and increase the Type-II error rate. Hedges' $g$ was calculated as a measure of the effect sizes based on the mean and standard deviations. This adaptation of the commonly reported Cohen's $d$ corrects for a bias due to small sample sizes (Hedges \& Olkin, 1985). For Dutch and English WRF, vocabulary and spelling, repeated measures ANOVAs with a $3 \times 2$ design were used to investigate if the effect of Group (LRnonDys, HRDys, HRnonDys) was larger in a certain marginally significant effects $(p<.1)$ that would be significant if we tested one-sided. If variables were not normally distributed, and/or if the homogeneity of variance assumption was violated we also ran non-parametric analyses, but since the conclusions based on the nonparametric analyses were very similar we only report the results of the parametric analyses.

\section{Missing Data Analysis and Descriptive Statistics}


were missing for the spoonerism reaction time due to test administration errors, registration or

360

361

362

363

364

365

366

367

368

369

370

371

372

373

374

375

376

377

378

379

380

computer errors. Since the data were missing completely at random $\left(\chi^{2}(35)=26.510, p=.848\right)$,

and the number of missing cases was small, missing cases were imputed with the expectation

maximization method in SPSS. Normality within the groups was checked with the Kolmogorov-

Smirnov test. In Table 2 with descriptive statistics it is indicated when the distribution deviated significantly from a normal distribution. The data of the Spoonerisms RT, which had a severe positive skewness and kurtosis, was successfully transformed with a log transformation; for the other variables that were not normally distributed transformations were not successful. There was homogeneity of variance for most variables, except for Digit-span - Forward, Spoonerisms Accuracy and RT, and Dutch spelling. To make the scales of the different reading and reading related measures comparable, the data were standardized on the basis of the means and standard deviation of the whole low familial risk group, including 4 participants with dyslexia. Therefore the means and standard deviations of the LRnonDys group slightly deviate from zero and one, respectively. Correlations between the different reading (related) measures can be found in Table 3. In table B1 in the appendix descriptive statistics and the minimum and maximum score can be found for the unstandardized data for all groups and for the total sample.

\section{Group Comparison}

The MANOVA revealed a significant overall effect of Group (Pillai's Trace $=.96, F(26$, 13) $=4.43, p<.001, \eta_{p}{ }^{2}=.48$ ). Pillai's Trace is reported because Box's test of equality of covariance matrices was significant $(M=346.64, F(282,14371.80)=1.42 p<.001)$. For all measures the effect of Group was significant $(p<.05)$. Pairwise comparisons with the Least Significant Difference method were performed after the MANOVA; effect sizes and 
381 significances can be found in Table 4. The HRDys group performed significantly worse than the

382 LRnonDys control group on all measures, and effect sizes were especially large for the reading

383 fluency and spelling measures. The HRDys group also performed significantly worse than the

384 HRnonDys group on most measures, except for the digit span forward task and Dutch

385 vocabulary, although the difference was marginally significant $(p=.07)$ for the latter test.

386 Although the HRnonDys group always scored in between the HRDys and LRnonDys group,

387 their performance was in most cases not significantly different from the LRnonDys group.

388 Exceptions here are the Dutch pseudoword reading fluency, spoonerisms RT and the digit-span

389 Forward tasks where the HRnonDys group performed significantly worse than the LRnonDys

390 group. For English spelling there was a marginally significant difference between the two non-

391 dyslexic groups $(p=.09)$.

392 Comparison between English and Dutch Literacy Skills

As can be seen from the correlations in Table 3, English and Dutch WRF and spelling

skills were overall strongly correlated, while there was only a small correlation between Dutch

and English vocabulary. To compare Dutch and English WRF, spelling and vocabulary between groups, we performed a 2x3 repeated measures ANOVAs with Language (English vs. Dutch) as

within-subject factor, and Group (LRnonDys vs. HRDys vs. HRnonDys) as between subject

factor. For WRF there was a significant main effect of $\operatorname{Group}(F(2,74)=35.09, p<.001$,

$\left.\eta_{p}{ }^{2}=.49\right)$, and a significant interaction between Language and Group $(F(2,74)=5.15, p=.01$,

$\left.\eta_{p}{ }^{2}=.12\right)$. There was no main effect of Language $\left(F(1,74)=.35, p=.56, \eta_{p}{ }^{2}=.01\right)$. A main effect

of Language could not be expected in this analysis, since the data were normalized per subtest and thus per language. Pairwise comparisons using the LSD method revealed that the HRDys group overall performed poorer than the LRNonDys and HRNonDys groups $(p<.05)$, but that 
404 the nonDys groups did not differ from each other $(p>.05)$. Further analyses of the interaction

405 showed that the difference between English and Dutch WRF (calculated by subtracting the

406 Dutch WRF score from the English WRF score) was larger for the HRDys group $(M=.44, S E=$ 407 .14) compared to the LRnonDys group $(M=-.03, S E=.14, p=.04)$ and HRnonDys group $(M=$ -

$408.26, S E=.18, p=.002$ ). Thus, the HRDys group performed relatively well on English compared

409 to Dutch WRF. In Figure 1, that shows the means and standard errors per group per language,

410 this interaction can also be observed. The difference between the LRnonDys and HRnonDys

411 groups was not significant $(p=.30)$.

412

There also was a main effect of Group on spelling $\left(F(2,74)=41.36, p>.001, \eta_{p}{ }^{2}=.53\right)$

413

414

415

416

417

418

and vocabulary $\left(F(2,74)=6.34, p=.003, \eta_{p}{ }^{2}=.15\right)$. Pairwise comparisons for both spelling and vocabulary revealed that the HRDys group performed poorer than the LRNonDys and HRNonDys groups $(p<.05)$, but that both nonDys groups did not differ from each other $(p>$ .05). In contrast to WRF, there was no interaction between Language and Group for spelling $(F$ $\left.(2,74)=1.58, p=.21, \eta_{p}{ }^{2}=.04\right)$ or vocabulary $\left(F(2,74)=.17, p=.84, \eta_{p}{ }^{2}=.005\right)$. There was also no main effect of Language on spelling $\left(F(1,74)=.1 .48, p=.23, \eta_{p}{ }^{2}=.02\right)$ or vocabulary $(F(1$, 74) $\left.=.01, p=.91, \eta_{p}{ }^{2}>.000\right)$, but again this was expected because the data were standardized per subtest.

\section{Discussion}

Reading Skills of Adolescents (with a Familial Risk of) Dyslexia

The first aim of this study was to investigate how adolescents with a familial risk of dyslexia perform at reading and spelling tasks in Dutch and ESL in comparison to a low familial risk control group without dyslexia. Moreover, we compared these groups on several literacy related measures: English and Dutch vocabulary, RAN, PA, and VST/WM. Large reading and 
427 spelling deficits and mild vocabulary deficits were found in the HRDys group, both in Dutch and

428 in English. The HRDys group scored significantly lower than the LRnonDys Group on all other

429 reading-related tasks as well. The HRDys group also scored significantly worse than the

430 HRnonDys group on all measures with the exception of Dutch vocabulary, where there was a

431 marginally significant difference. These findings are in agreement with previous studies that also

432 found that dyslexia is persistent into adolescence (Ferrer et al., 2015; Shaywitz et al., 1999).

433 We observed a three-step pattern in the data as the scores of the HRnonDys group were in

434 between the scores of the LRnonDys group and the scores of the HRDys group, but the

435 difference between the two nonDys groups was only significant for Dutch pseudoword reading,

436 the Spoonerisms RTs measuring Dutch PA and the digit span-forward task measuring VSTM.

437 The results are in line with studies of adolescents with a familial risk where the HRnonDys group

438 generally performs poorer than the LRnonDys group, but only some of the differences were

439 significant (Dandache et al., 2014; Eklund et al., 2014; Snowling et al., 2007). These results

440 support a continuous view of (the familial risk of) dyslexia and a multifactorial origin of dyslexia

441 as described by among others Pennington et al. (2012) and van Bergen et al. (2014). It is likely

442 that adolescents in the HRnonDys group have been exposed to some of the same family risk

443 factors that affected the HRDys group. However, based on this study we cannot determine

444 whether these family influences are genetic, environmental, both, or an interaction between

445 genetics and the environment. This view of dyslexia is further supported by family-risk studies

446 where the reading skills of parents of high FR children who did develop dyslexia have been

447 found to be even lower than the reading skills of parents of high FR children who did not

448 develop dyslexia (Torppa, Eklund, van Bergen, \& Lyytinen, 2011; van Bergen et al., 2011, 449 2012). 

compared to the nondyslexic groups, effect sizes were especially large for reading and spelling

452

453

measures, as would be expected based on the definition of dyslexia. For the reading related measures, effect sizes were especially large for Dutch PA, and in particular for the reaction times. Also, the largest difference between the HRnonDys and LRnonDys group was on this PA measure. These findings are in line with a phonological core deficit in dyslexia (Bekebrede et al., 2009; van der Leij \& Morfidi, 2006). Although it has been shown that phonological problems are neither necessary nor sufficient to explain dyslexia (Pennington et al., 2012), many children with dyslexia do have a severe phonological deficit. Our study suggests that also non-dyslexic adolescents with a high FR have phonological problems, measured with Dutch PA, although milder than students with dyslexia. While the HRnonDys group generally scored lower than the LRnonDys group, their RAN, Verbal Working Memory (VWM) and both English and Dutch vocabulary scores were very similar, as indicated by the small effect sizes. For the HRnonDys group RAN, VWM and/or vocabulary knowledge may be protective factors or compensations for their phonological deficit; further research is needed to investigate whether the good RAN, VWM and vocabulary skills are a cause or consequence of their reading level, or whether they are merely correlated. These results are in line with Moll, Loff and Snowling (2013) who found that PA and VSTM were associated with family risk status as well as with dyslexia status. The authors argued that phonological skills can be considered as a cognitive endophenotype of dyslexia. In addition, RAN, word recall and morphological awareness were also in their study only associated with dyslexia status, and were seen as additional risk factors for dyslexia. For both HR groups effect sizes were largest for pseudoword reading in comparison to the other reading and spelling tasks, probably because pseudoword reading relies mainly on letter-to- 
473 phoneme decoding, in addition to the use of sub-lexical orthographic units, but word-based

474 orthographic strategies cannot be used to compensate for their phonological deficit.

\section{English Versus Dutch}

476 Our second aim was to make a direct comparison of reading, spelling and vocabulary

477 skills in Dutch and English. Overall Dutch and English reading, spelling and vocabulary skills

478 were significantly correlated; there were strong positive relationships between L1 and L2 reading

479 and spelling, and small positive relationship between L1 and L2 vocabulary. Thus, adolescents

480 with dyslexia had not only a literacy impairment in Dutch, but generally also in ESL. These

481 findings are in agreement with the LCDH (Ganschow et al., 1991) which states that L1 reading

482 skills are a strong predictor for L2 reading outcomes. Furthermore, previous studies have also

483 observed reading problems in L2 among readers with a reading deficit in L1 (e.g. Helland \&

484 Kaasa, 2005; Helland \& Morken, 2016; Łockiewicz \& Jaskulska, 2016).

485 To our knowledge, our study was the first to make a direct comparison of Dutch and ESL

486 in a group of adolescents with a familial risk of dyslexia. This did not only allow us to study

487 whether deficits of the dyslexic group were similar across languages, but also whether the

488 HRnonDys group, which by definition has no reading deficit in L1, has literacy problems in L2.

489 We found that the HRDys group performed, relative to the LRnonDys control group, better on

490 WRF in English compared to Dutch, however, this interaction effect was small; for spelling and

491 vocabulary this effect was not found. The HRnonDys group did not show any significant

492 differences between Dutch and English reading, spelling and vocabulary, as compared to this

493 LRnonDys pattern. The relatively greater reading fluency in English than in Dutch in the HRDys

494 group may seem surprising as the lower orthographic transparency of English makes reading in

495 English more difficult, at least for beginning readers (Seymour et al., 2003). Moreover, the 
496

497

498

499

500

501

502

503

504

505

506

507

508

509

510

511

512

513

514

515

516

517

518

adolescents in our study were unbalanced bilinguals who acquired English later than Dutch, and who were generally more proficient in Dutch, therefore they may be less able to compensate using semantics-based reading strategies.

However, other factors may be involved as well. Because our participants were not beginning readers in Dutch, they had presumably already developed a whole-word based reading strategy in Dutch, and perhaps they could already apply this strategy when they learned to read in English. Because of the lower orthographic transparency, whole-word reading is more successful than decoding in English. Perhaps the reading deficit of dyslexic readers in ESL was therefore smaller than expected based on their Dutch reading. Furthermore, not all readers with dyslexia have an orthographic processing deficit (van der Leij \& Morfidi, 2006). In fact, an earlier study by Siegel, Share and Geva (1995) found that English dyslexic readers had superior orthographic skills compared to normal readers, which may suggest that they rely more on the visual orthographic characteristics of a word than on its phonological characteristics. This result has, however, not been replicated. It is unclear whether the dyslexics as a group show better orthographic skills, or that it is a characteristic for a substantial subgroup, as is predicted by the phonological-core variable-orthographic differences model (e.g. Bekebrede et a., 2009). To read successfully in English, a reader needs to identify larger orthographic units than in Dutch. Therefore having average to good orthographic skills is more beneficial for reading in English.

Some readers with dyslexia may have been able to use orthographic strategies to compensate for their phonological deficit in ESL, like the readers with a preference for English in the study by Miller-Guron and Lundberg (2000).

Another influencing factor responsible for the advantage of ESL for students with dyslexia relates to the massive exposure to English that (young) people have nowadays. As the 
519 use of English becomes more and more important for adolescents to participate in modern

520 society, the motivation to learn English may be high. Perhaps reading in English is also less

521 associated with the frustration individuals with reading difficulties experienced as beginning

522 readers in Dutch, because, when they learned ESL, they had already developed some

523 compensation strategies in Dutch. These hypotheses are still speculative and need further

524 investigation, but they seem to be supported by a few recent studies. In a Norwegian study by

525 Brevik, Olson and Hellekjaer (2016) it was found that 22 percent of the worst readers in

526 Norwegian L1 had good reading skills in ESL. Most of these poor L1 but good L2 readers were

527 boys in vocational education programs. A follow-up case study of five boys in vocational

528 programs with poor L1 Norwegian reading skills but good ESL reading skills showed that they

529 all played online games in English and also used English more than Norwegian for Facebook, 530 music, TV and films (Brevik, 2016). Also Sundqvist and Wikström (2015) found that frequent

531 online-gamers, who were again mostly boys, had the highest English grades, wrote better English 532 essays in which they used more advanced vocabulary, and had a larger L2 vocabulary size. High

533 exposure through multimedia and intrinsic motivation to learn English as a lingua franca in

534 modern society are two possible explanations for the slight advantage we found in English for

535 students with dyslexia compared to their mother tongue.

536 Limitations and Future Directions

537 In the present study we have chosen to use a categorical approach towards dyslexia and

538 familial risk. We have done so because categorical diagnoses are still used for clinical practice

539 and for the comparability with other studies. However we do realize that both dyslexia and

540 familial risk are continuous constructs as we have explained in the introduction. Because of the

541 longitudinal design groups were not matched and we had no control over factors like the quantity 
542 and quality of the remediation, instruction in and exposure to English, and had only limited

543 information available about these factors. Future cross-sectional studies could look further into

544 these factors as they could be relevant in the context of our research question. Since this study

545 was conducted at the end of a longitudinal design, it had a higher dropout rate than previous

546 waves of the DDP program, leaving the groups smaller than we had hoped for. This affected the

547 statistical power, and may be the reason why some of the small effects between the two non-

548 dyslexic groups were not significant. However, the present study indicates that the differences

549 between the HRnonDys and LRnonDys groups are not large. For the comparison of Dutch and

550 English vocabulary it would have been better if we used more similar tests. Because of time

551 concerns, we used the vocabulary test from the WISC-III-NL (Wechsler, 2005) for Dutch and the

552 more elaborate PPVT-IV (Dunn \& Dunn, 2007) for English. This could at least partially explain

553 why the correlations between the English and Dutch vocabulary tests were small. Moreover, a

554 standardized spelling ESL test for Dutch learners was not available. So, we had to create a new

555 test. However, because of strong correlations with the Dutch spelling test and the English WRF,

556 we judged it sufficiently valid and reliable.

557 Including a larger sample would allow for the investigation of factors that contribute

558 stronger to reading in Dutch versus reading in English, and whether this is the same for groups

559 with a different risk and dyslexia status. Moreover, this would allow us to look at subgroups, or

560 even at the individual level to investigate the influence of exposure and motivation in more

561 detail. In the present study we did not look at orthographic processing, but it would be really

562 interesting to include this in future studies, and to also consider higher level reading skills such

563 as sentence and text comprehension, silent reading, and to measure reading related skills and

564 pseudoword reading also in L2. Future studies could also use neuro-imaging techniques to 
565 investigate which brain networks are involved in reading in L1 and L2 in the different groups.

566 Such research could also help to identify compensation mechanisms used by the HRnonDys

567 group that allows them to read well despite the mild phonological processing difficulties that we

568 found in the present study using the Dutch PA task. Knowing more about what contributes to

569 reading success in ESL is not only important for theoretical purposes but could also be useful for

570 future interventions.

\section{Conclusions and Implications}

572 We found that dyslexia is persistent in adolescents with a high familial risk of dyslexia.

573 Although the reading deficit of the adolescents with dyslexia was slightly smaller in ESL than

574 expected based on their Dutch WRF skills, adolescents with dyslexia generally also have large

575 reading and spelling deficits in L2. Therefore it is important that they still receive help for this in

576 secondary education and are granted access to measures like extra time in exams and the use of

577 spellcheckers in L1 and L2. A phonological deficit was found in both groups with a familial risk,

578 however it was less severe in the HRnonDys group who may also have used their better RAN-

579 and VWM-abilities to compensate. Despite their subtle phonological deficit, and the low

580 orthographic transparency of English, the HRnonDys group did not have reading difficulties in

581 ESL. Thus, their familial risk seems to affect L1 and L2 in a similar way, and extra help in ESL

582 is probably not needed for most of these students. 


\section{References}

584

585

586

587

588

589

590

591

592

593

594

595

596

597

598

599

600

601

602

603

604

American Psychiatric Association. (2013). Diagnostic and statistical manual of mental disorders: DSM-5. Washington, D.C: American Psychiatric Association.

Bekebrede, J., van der Leij, A., \& Share, D. L. (2009). Dutch dyslexic adolescents: phonologicalcore variable-orthographic differences. Reading and Writing, 22(2), 133-165. https://doi.org/10.1007/s11145-007-9105-7

Brevik, L. M. (2016). The Gaming Outliers: Does out-of-school gaming improve boys' reading skills in English as a second language. In E. Elstad (Ed.), Educational Technology and Polycontextual Bridging (in Elstad, E. (Ed.), pp. 39-61). Rotterdam, the Netherlands: Sense Publishers.

Brevik, L. M., Olsen, R. V., \& Hellekjær, G. O. (2016). The complexity of second language reading: Investigating the L1-L2 relationship. Reading in a Foreign Language, 28(2), 2.

Brus, B. T., \& Voeten, M. J. M. (1973). Een minuut test, vorm A en B. Verantwoording en handleiding. Nijmegen: Berkhout.

Carrion-Castillo, A., Franke, B., \& Fisher, S. E. (2013). Molecular genetics of dyslexia: An overview. Dyslexia, 19(4), 214-240. https://doi.org/10.1002/dys.1464

Carrion-Castillo, A., Maassen, B., Franke, B., Heister, A., Naber, M., van der Leij, A., ... Fisher, S. E. (2017). Association analysis of dyslexia candidate genes in a Dutch longitudinal sample. European Journal of Human Genetics. https://doi.org/10.1038/ejhg.2016.194

d'Ydewalle, G., \& Poel, M. V. de. (1999). Incidental foreign-language acquisition by children watching subtitled television programs. Journal of Psycholinguistic Research, 28(3), 227-244. https://doi.org/10.1023/A:1023202130625 
605 Dandache, S., Wouters, J., \& Ghesquière, P. (2014). Development of reading and phonological 606 skills of children at family risk for dyslexia: A longitudinal analysis from kindergarten to 607 sixth grade. Dyslexia, 20(4), 305-329. https://doi.org/10.1002/dys.1482

608

609

610

611

612

613

614

615

616

617

618

619

620

621

622

623

624

625

626 Experimental Child Psychology, 70(2), 75-96. https://doi.org/10.1006/jecp.1998.2451

Depessemier, P., \& Andries, C. (2009). Gl\&schr. Test voor gevorderd Lezen en Schrijven. Antwerpen: Garant.

Dewey, G. (1971). English spelling: Roadblock to reading. New York: Teachers College Press.

Di Carlo, A. (1994). Comprehensible Input through the Practical Application of Video-Texts in Second Language Acquisition. Italica, 71(4), 465-483. https://doi.org/10.2307/479666

Dunn, L. M., \& Dunn, D. M. (2007). Peabody Picture Vocabulary Test (4th ed.). San Antonio, TX: Pearson.

Eklund, K., Torppa, M., Aro, M., Leppänen, P. H. T., \& Lyytinen, H. (2014). Literacy skill development of children with familial risk for dyslexia through grades 2,3 , and 8 . Journal of Educational Psychology. https://doi.org/10.1037/a0037121

Elbro, C., Borstrøm, I., \& Petersen, D. K. (1998). Predicting dyslexia from kindergarten: The importance of distinctness of phonological representations of lexical items. Reading Research Quarterly, 33(1), 36-60. https://doi.org/10.1598/RRQ.33.1.3

Ferrer, E., Shaywitz, B. A., Holahan, J. M., Marchione, K. E., Michaels, R., \& Shaywitz, S. E. (2015). Achievement gap in reading is present as early as first grade and persists through adolescence. The Journal of Pediatrics, 167(5), 1121-1125.e2. https://doi.org/10.1016/j.jpeds.2015.07.045 
627 Ganschow, L., Sparks, R. L., Javorsky, J., Pohlman, J., \& Bishop-Marbury, A. (1991).

628

629

630

631

632

633

634

635

636

637

638

639

640

641

642

643

644

645

646

647

648

649

Identifying native language difficulties among foreign language learners in college: A “foreign” language learning disability? Journal of Learning Disabilities, 24(9), 530-541. https://doi.org/10.1177/002221949102400905

Geelhoed, J., \& Reitsma, P. (2004). PI-Signaleringsdictee voor brugklassen. Amsterdam: Harcourt.

Georgiou, G. K., Papadopoulos, T. C., Zarouna, E., \& Parrila, R. (2012). Are auditory and visual processing deficits related to developmental dyslexia? Dyslexia, 18(2), 110-129. https://doi.org/10.1002/dys.1439

Germanò, E., Gagliano, A., \& Curatolo, P. (2010). Comorbidity of ADHD and dyslexia. Developmental Neuropsychology, 35(5), 475-493. https://doi.org/10.1080/87565641.2010.494748

Gerritsen, M., Van Meurs, F., Planken, B., \& Korzilius, H. (2016). A reconsideration of the status of English in the Netherlands within the Kachruvian Three Circles model. World Englishes, 35(3), 457-474. https://doi.org/10.1111/weng.12206

Hagtvet, B. E., \& Lyster, S.-A. H. (2003). The spelling errors of Norwegian good and poor decoders: A developmental cross-linguistic perspective. In Dyslexia in different languages: Cross-linguistic comparisons (pp. 181-207). Philadelphia, PA, US: Whurr Publishers.

Hedges, L. V., \& Olkin, I. (1985). Statistical methods for meta-analysis. New York: Academic Press.

Helland, T., \& Kaasa, R. (2005). Dyslexia in English as a second language. Dyslexia, 11(1), 4160. https://doi.org/10.1002/dys.286 
650 Helland, T., \& Morken, F. (2016). Neurocognitive development and predictors of L1 and L2 651 literacy skills in dyslexia: A longitudinal study of children 5-11 years old. Dyslexia, 652 22(1), 3-26. https://doi.org/10.1002/dys.1515

653 Kaplan, B. J., Wilson, B. N., Dewey, D., \& Crawford, S. G. (1998). DCD may not be a discrete 654 disorder. Human Movement Science, 17(4-5), 471-490. https://doi.org/10.1016/S0167-

655 9457(98)00010-4

656 Kirby, J. R., Georgiou, G. K., Martinussen, R., \& Parrila, R. (2010). Naming speed and reading:

657

658

659

660

661

662

663

664

665

666

667

668

669

670

671

672 From prediction to instruction. Reading Research Quarterly, 45(3), 341-362. https://doi.org/10.1598/RRQ.45.3.4

Kleijnen, R., Steenbeek-Planting, E., \& Verhoeven, L. (2008). Toetsen en Interventies bij Dyslexie in het Voortgezet Onderwijs, Nederlands en de Moderne Vreemde Talen. Nijmegen: Expertise Centrum Nederlands.

Kuijpers, C., van der Leij, A., Been, P., van Leeuwen, T., ter Keurs, M., Schreuder, R., \& van den Bos, K. P. (2003). Leesproblemen in de bovenbouw van het voortgezet onderwijs en de volwassenheid: Normering van een aantal tests. Pedagogische Studiën, 80, 272-287.

Łockiewicz, M., \& Jaskulska, M. (2016). Difficulties of Polish students with dyslexia in reading and spelling in English as L2. Learning and Individual Differences, 51, 256-264. https://doi.org/10.1016/j.lindif.2016.08.037

Mayes, S. D., \& Calhoun, S. L. (2006). Frequency of reading, math, and writing disabilities in children with clinical disorders. Learning and Individual Differences, 16(2), 145-157. https://doi.org/10.1016/j.lindif.2005.07.004

McArthur, T. (1996). English in the world and in Europe. In R. R. Hartmann (Ed.), The English language in Europe (pp. 3-15). Oxford: Intellect. 
673 Miles, T. R. (2004). Some problems in determining the prevalence of dyslexia. Electronic

674

675

676

677

678

679

680

681

682

683

684

685

686

687

688

689

690

691

692

693

694

Journal of Research in Educational Psychology, 2(2), 5-12.

Miller-Guron, L., \& Lundberg, I. (2000). Dyslexia and second language reading: A second bite at the apple? Reading and Writing, 12(1-2), 41-61.

https://doi.org/10.1023/A:1008009703641

Moll, K., Loff, A., \& Snowling, M. J. (2013). Cognitive Endophenotypes of Dyslexia. Scientific Studies of Reading, 17(6), 385-397. https://doi.org/10.1080/10888438.2012.736439

Norton, E. S., \& Wolf, M. (2012). Rapid automatized naming (RAN) and reading fluency: Implications for understanding and treatment of reading disabilities. Annual Review of Psychology, 63(1), 427-452. https://doi.org/10.1146/annurev-psych-120710-100431

Papadopoulos, T. C., Spanoudis, G. C., \& Georgiou, G. K. (2016). How is RAN related to reading fluency? A comprehensive examination of the prominent theoretical accounts. Educational Psychology, 1217. https://doi.org/10.3389/fpsyg.2016.01217

Pennington, B. F. (2006). From single to multiple deficit models of developmental disorders. Cognition, 101(2), 385-413. https://doi.org/10.1016/j.cognition.2006.04.008

Pennington, B. F., \& Lefly, D. L. (2001). Early reading development in children at family risk for dyslexia. Child Development, 72(3), 816-833. https://doi.org/10.1111/14678624.00317

Pennington, B. F., Santerre-Lemmon, L., Rosenberg, J., MacDonald, B., Boada, R., Friend, A., ... Olson, R. K. (2012). Individual prediction of dyslexia by single vs. multiple deficit models. Journal of Abnormal Psychology, 121(1), 212-224.

https://doi.org/10.1037/a0025823 
695 Ramus, F., Rosen, S., Dakin, S. C., Day, B. L., Castellote, J. M., White, S., \& Frith, U. (2003).

696 Theories of developmental dyslexia: Insights from a multiple case study of dyslexic

697 adults. Brain, 126(4), 841-865. https://doi.org/10.1093/brain/awg076

698

699

700

701

702

703

704

705

706

707

708

709

710

711

712

713

714

715

716

717

Rothe, J., Cornell, S., Ise, E., \& Schulte-Körne, G. (2015). A comparison of orthographic processing in children with and without reading and spelling disorder in a regular orthography. Reading and Writing, 28(9), 1307-1332. https://doi.org/10.1007/s11145$015-9572-1$

Schijf, G. M. (2009). Lees- en spellingvaardigheden van brugklassers. University of Amsterdam. Retrieved from http://hdl.handle.net/11245/1.316956

Seymour, P. H. K., Aro, M., \& Erskine, J. M. (2003). Foundation literacy acquisition in European orthographies. British Journal of Psychology, 94(2), 143-174. https://doi.org/10.1348/000712603321661859

Shaywitz, S. E., Escobar, M. D., Shaywitz, B. A., Fletcher, J. M., \& Makuch, R. (1992). Evidence that dyslexia may represent the lower tail of a normal distribution of reading ability. New England Journal of Medicine, 326(3), 145-150. https://doi.org/10.1056/NEJM199201163260301

Shaywitz, S. E., Fletcher, J. M., Holahan, J. M., Shneider, A. E., Marchione, K. E., Stuebing, K. K., ... Shaywitz, B. A. (1999). Persistence of dyslexia: The Connecticut longitudinal study at adolescence. Pediatrics, 104(6), 1351-1359. https://doi.org/10.1542/peds.104.6.1351

Siegel, L. S., Share, D., \& Geva, E. (1995). Evidence for Superior Orthographic Skills in Dyslexics. Psychological Science, 6(4), 250-254. https://doi.org/10.1111/j.14679280.1995.tb00601.x 
718 Snowling, M. J., Gallagher, A., \& Frith, U. (2003). Family risk of dyslexia is continuous:

719 Individual differences in the precursors of reading skill. Child Development, 74(2), 358-

720 373. https://doi.org/10.1111/1467-8624.7402003

721

722

723

724

725

726

727

728

729

730

731

732

733

734

735

736

737

738

739

740

Snowling, M. J., \& Melby-Lervåg, M. (2016). Oral language deficits in familial dyslexia: A meta-analysis and review. Psychological Bulletin, 142(5), 498-545. https://doi.org/10.1037/bul0000037

Snowling, M. J., Muter, V., \& Carroll, J. (2007). Children at family risk of dyslexia: A follow-up in early adolescence. Journal of Child Psychology and Psychiatry, 48(6), 609-618. https://doi.org/10.1111/j.1469-7610.2006.01725.x

Sundqvist, P., \& Wikström, P. (2015). Out-of-school digital gameplay and in-school L2 English vocabulary outcomes. System, 51, 65-76. https://doi.org/10.1016/j.system.2015.04.001

Thijs, A., Trimbos, B., Tuin, D., Bodde, M., \& de graaff, R. (2011). Engels in het basisonderwijs - vakdossier - SLO, nationaal expertisecentrum leerplanontwikkeling. Retrieved November 14, 2016, from http://www.slo.nl/downloads/2011/engels-in-hetbasisonderwijs-vakdossier.pdf/

Tops, W., Callens, C., Cauwenberghe, E. V., Adriaens, J., \& Brysbaert, M. (2013). Beyond spelling: the writing skills of students with dyslexia in higher education. Reading and Writing, 26(5), 705-720. https://doi.org/10.1007/s11145-012-9387-2

Torppa, M., Eklund, K., van Bergen, E., \& Lyytinen, H. (2011). Parental literacy predicts children's literacy: A longitudinal family-risk study. Dyslexia, 17(4), 339-355. https://doi.org/10.1002/dys.437

van Bergen, E., de Jong, P. F., Plakas, A., Maassen, B. A. M., \& van der Leij, A. (2012). Child and parental literacy levels within families with a history of dyslexia. Journal of Child 
741

742

743

744

745

746

747

748

749

750

751

752

753

754

755

756

757

758

759

760

761

762

Psychology and Psychiatry, 53(1), 28-36. https://doi.org/10.1111/j.14697610.2011.02418.x

van Bergen, E., de Jong, P. F., Regtvoort, A., Oort, F., van Otterloo, S., \& van der Leij, A. (2011). Dutch children at family risk of dyslexia: precursors, reading development, and parental effects. Dyslexia, 17(1), 2-18. https://doi.org/10.1002/dys.423

van Bergen, E., van der Leij, A., \& de Jong, P. F. (2014). The intergenerational multiple deficit model and the case of dyslexia. Frontiers in Human Neuroscience, 8. https://doi.org/10.3389/fnhum.2014.00346

van den Bos, K. P., \& Lutje Spelberg, H. C. (2007). Continu Benoemen \& Woorden Lezen. Een test voor het diagnosticeren van taal- en leesstoornissen. Handleiding. Amsterdam: Boom Testuitgevers.

van den Bos, K. P., Lutje Spelberg, H. C., Scheepstra, A. J. M., \& de Vries, J. R. (1994). De Klepel vorm A en B. Een test voor de leesvaardigheid van pseudowoorden. Verantwoording, handleiding, diagnostiek en behandeling. Nijmegen: Berkhout.

van der Leij, A., \& Morfidi, E. (2006). Core deficits and variable differences in Dutch poor readers learning English. Journal of Learning Disabilities, 39(1), 74-90. https://doi.org/10.1177/00222194060390010701

van der Leij, A., van Bergen, E., van Zuijen, T., de Jong, P. F., Maurits, N. M., \& Maassen, B. A. M. (2013). Precursors of developmental dyslexia: An overview of the longitudinal Dutch dyslexia programme study. Dyslexia, 19(4), 191-213. https://doi.org/10.1002/dys.1463

Wechsler, D. (2005). Wechsler Intelligence Scale for Children, Dutch Version. (3rd ed.). Amsterdam: Pearson. 
763 Willcutt, E. G., \& Pennington, B. F. (2000). Comorbidity of reading disability and attention764 deficit/hyperactivity disorder: Differences by gender and subtype. Journal of Learning 765 Disabilities, 33(2), 179-191. https://doi.org/10.1177/002221940003300206 


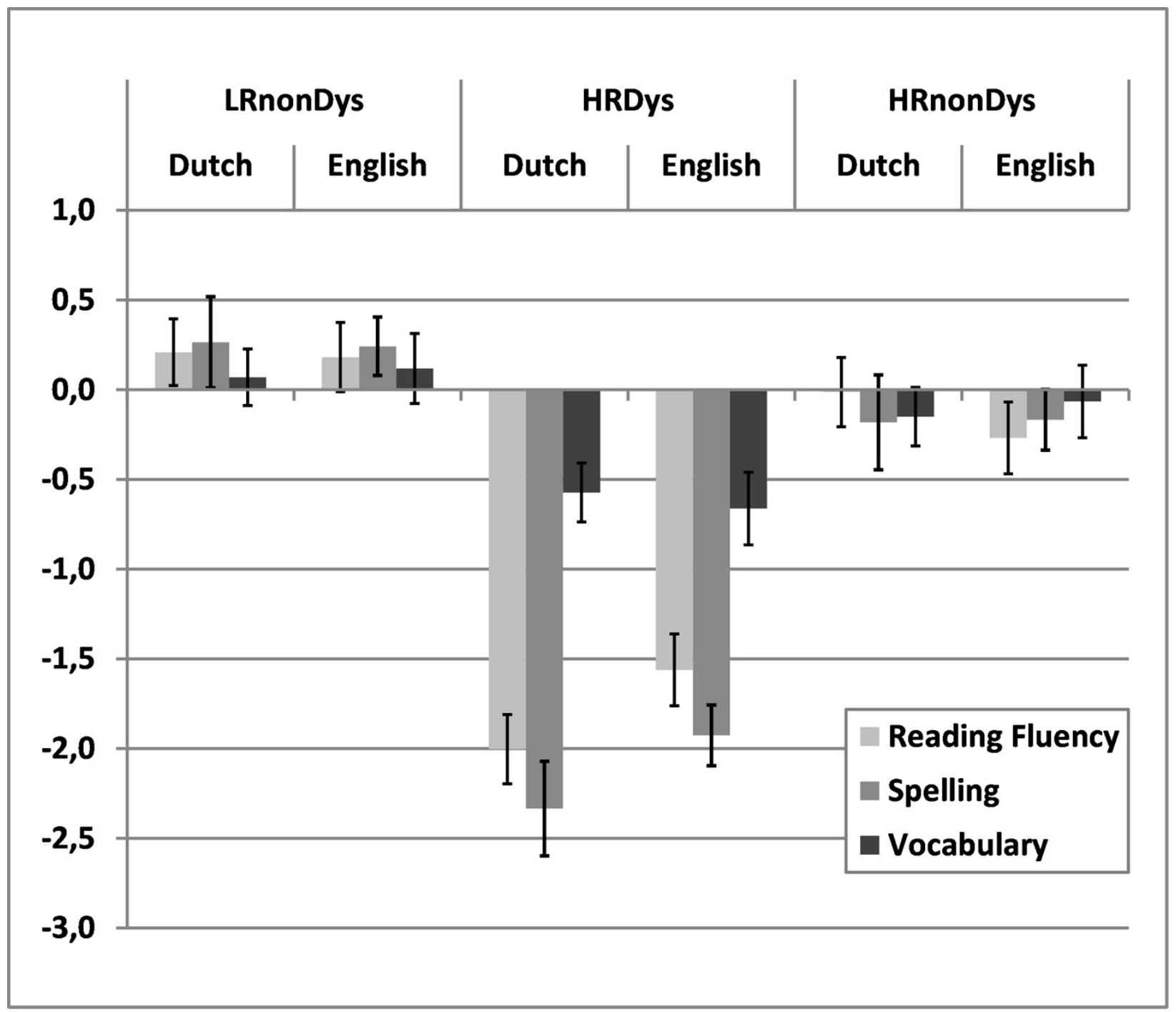

Figure 1.

Mean Standardized Scores on Dutch and English Word Reading Fluency, Spelling and Vocabulary for the Three Groups. Error Bars indicate Standard Errors. (LRnonDys = low familial risk without dyslexia, HRnonDys = high familial risk without dyslexia, HRDys = high familial risk with dyslexia) 
Table 1.

Participant Characteristics

\begin{tabular}{|c|c|c|c|c|c|c|}
\hline \multirow{2}{*}{ Group } & \multirow{2}{*}{$n$} & \multirow{2}{*}{$n$ males } & \multicolumn{2}{|c|}{ Age (Months) } & \multicolumn{2}{|c|}{ School Grade } \\
\hline & & & $M$ & $S D$ & $M$ & $S D$ \\
\hline LRnonDys & 27 & 11 & 166.9 & 5.3 & 8.1 & .5 \\
\hline LRDys & 4 & 2 & 163.5 & 4.4 & 7.8 & .5 \\
\hline HRnonDys & 25 & 12 & 170.0 & 9.0 & 8.1 & .7 \\
\hline HRDys & 25 & 14 & 170.7 & 6.8 & 8.1 & .9 \\
\hline
\end{tabular}

Note. LRnonDys $=$ Low Risk without Dyslexia, LRDys $=$ Low Risk with Dyslexia, HRnonDys $=$ High Risk without Dyslexia, HRDys $=$ High Risk with Dyslexia. 
Table 2.

Descriptive Statistics of Standardized Scores per Group

\begin{tabular}{|c|c|c|c|c|c|c|c|c|c|}
\hline & \multicolumn{3}{|c|}{$\begin{array}{c}\text { LRnonDys } \\
(n=27)\end{array}$} & \multicolumn{3}{|c|}{$\begin{array}{c}\text { HRnonDys } \\
(n=25)\end{array}$} & \multicolumn{3}{|c|}{$\begin{array}{l}\text { HRDys } \\
(n=25)\end{array}$} \\
\hline & $M(S D)$ & Skewness & Kurtosis & $M(S D)$ & Skewness & Kurtosis & $M(S D)$ & Skewness & Kurtosis \\
\hline Dutch Words RF & $.21(.78)$ & -.08 & -1.10 & $-.01(.96)$ & .11 & .14 & $-2.00(1.14)$ & -.76 & .25 \\
\hline Pseudoword RF & $.24(.80)$ & .06 & -.62 & $-.38(.83)$ & -.37 & .66 & $-1.75(.67)$ & -.95 & .71 \\
\hline Loanword RF & $.21(.85)$ & -.31 & .35 & $-.21(.84)$ & .37 & 1.34 & $-1.77(1.52)$ & -.42 & .18 \\
\hline English Word RF & $.18(.87)$ & .08 & -.61 & $-.27(.83)$ & .66 & 1.53 & $-1.56(1.25)$ & .03 & .06 \\
\hline Dutch Spelling & $.26(.74)$ & -.62 & -.13 & $-.18(.99)$ & -.58 & -.35 & $-2.33(1.94)^{\mathrm{a}}$ & -.39 & -1.18 \\
\hline English Spelling & $.24(.79)$ & -1.19 & 1.99 & $-.17(.77)^{\mathrm{a}}$ & -1.48 & 2.71 & $-1.93(.97)^{\mathrm{a}}$ & .29 & -.94 \\
\hline Dutch Vocabulary & $.07(1.03)$ & -2.22 & 7.33 & $-.15(.62)$ & .36 & -.23 & $-.57(.73)$ & -.66 & -.44 \\
\hline English Vocabulary & $.12(.98)$ & -.41 & -.39 & $-.07(.85)$ & -.69 & .24 & $-.66(1.17)$ & -.10 & -.69 \\
\hline Spoonerisms - Acc & $.16(.59)^{\mathrm{a}}$ & -.71 & -.67 & $-.55(1.40)^{\mathrm{a}}$ & -1.19 & .41 & $-2.79(2.39)$ & -.59 & .99 \\
\hline Spoonerisms - RT (Log) & $-.27(.67)$ & .73 & .57 & $.66(.88)$ & -.56 & -.62 & $1.98(.91)$ & .73 & .74 \\
\hline RAN (Items / min) & $.18(.94)$ & .43 & -.56 & $-.01(.95)^{\mathrm{a}}$ & .75 & 2.19 & $-.58(.60)$ & -.24 & -.42 \\
\hline Digit Span - Forward & $.11(1.00)^{\mathrm{a}}$ & .15 & -1.14 & $-.56(.74)^{\mathrm{a}}$ & -.44 & -.27 & $-.95(.69)$ & .20 & -.78 \\
\hline Digit Span - Backward & $.15(.98)$ & -.01 & -.92 & $-.21(1.09)$ & .26 & .18 & $-.75(.75)^{\mathrm{a}}$ & -.20 & -1.10 \\
\hline
\end{tabular}

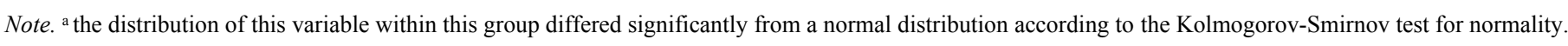

LRnonDys $=$ Low Risk without Dyslexia, HRnonDys $=$ High Risk without Dyslexia, HRDys $=$ High Risk with Dyslexia, RF $=$ Reading Fluency, Acc $=$ Accuracy, RT $=$ Reaction Time, RAN $=$ Rapid Automatized Naming. 
Table 3.

Pearson Correlation between Dutch and English Reading and Reading Related Skills for Participants in the LRnonDys, HRnonDys and HRDys group.

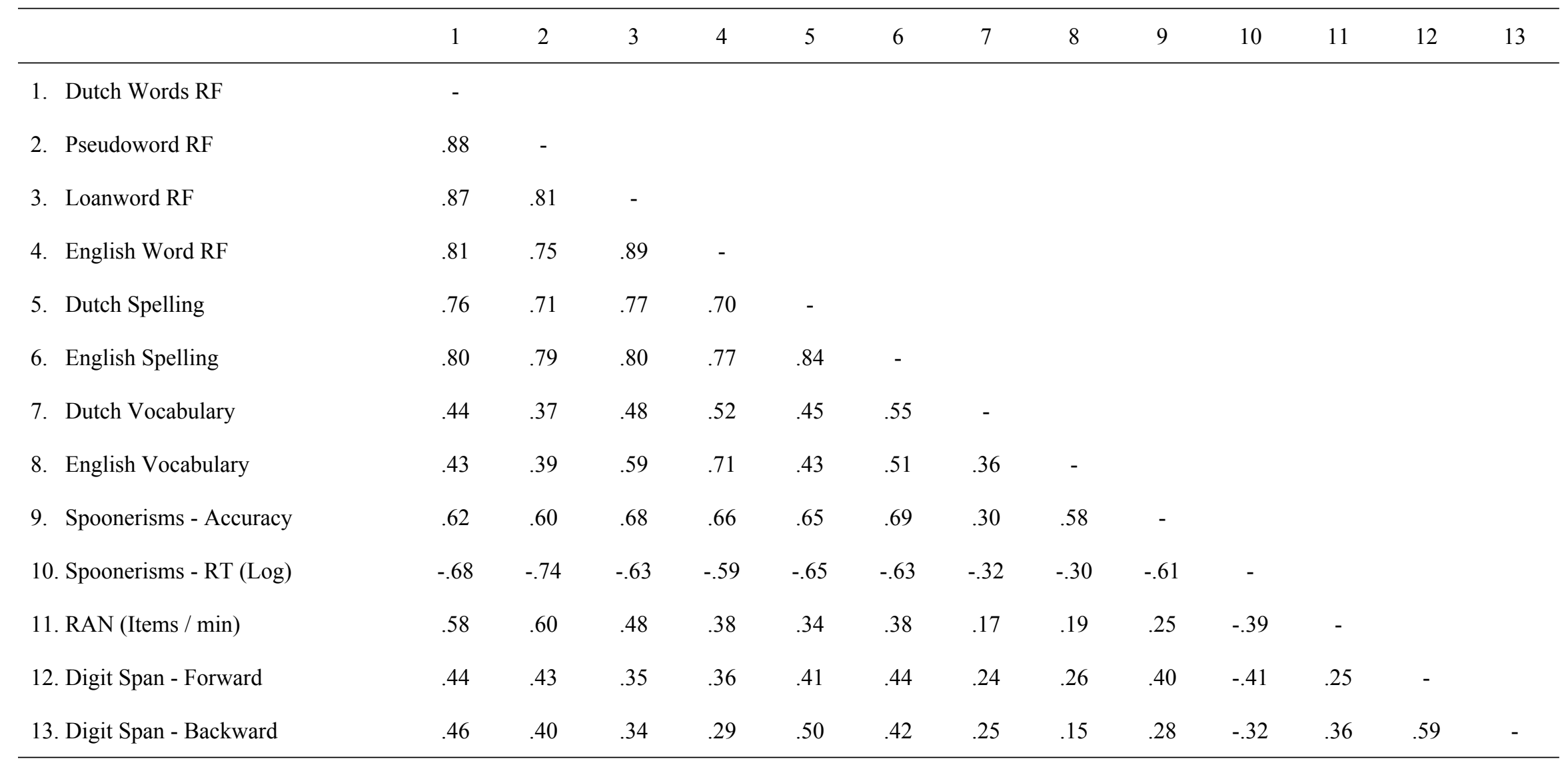

Note. $N=$ 77. LRnonDys $=$ Low Risk without Dyslexia, HRnonDys $=$ High Risk without Dyslexia, HRDys $=$ High Risk with Dyslexia, RF $=$ Reading Fluency, $\mathrm{RT}=$ Reaction Time, RAN $=$ Rapid Automatized Naming. Correlations of .19 and larger are significant at .1 level (2-tailed), Correlations of .24 and larger are significant at the .05 level (2-tailed), and correlations of .30 and larger are significant at the .01 level (2-tailed) 
Table 4.

Effect Sizes and Confidence Intervals of Group Differences.

\begin{tabular}{|c|c|c|c|c|c|c|c|c|c|}
\hline \multirow{4}{*}{ Measure } & \multicolumn{3}{|c|}{ LRnonDys - HRDys } & \multicolumn{3}{|c|}{ HRnonDys - HRDys } & \multicolumn{3}{|c|}{ LRnonDys - HRnonDys } \\
\hline & \multirow[b]{3}{*}{ Hedges' $g$} & \multirow{2}{*}{\multicolumn{2}{|c|}{$\begin{array}{c}95 \% \\
\text { Confidence Interval }\end{array}$}} & \multirow[b]{3}{*}{ Hedges' $g$} & \multirow{2}{*}{\multicolumn{2}{|c|}{$\begin{array}{c}95 \% \\
\text { Confidence Interval }\end{array}$}} & \multirow[b]{3}{*}{ Hedges' $g$} & \multirow{2}{*}{\multicolumn{2}{|c|}{$\begin{array}{c}95 \% \\
\text { Confidence Interval }\end{array}$}} \\
\hline & & & & & & & & & \\
\hline & & $\begin{array}{l}\text { Lower } \\
\text { bound }\end{array}$ & $\begin{array}{l}\text { Upper } \\
\text { bound }\end{array}$ & & $\begin{array}{l}\text { Lower } \\
\text { bound }\end{array}$ & $\begin{array}{l}\text { Upper } \\
\text { bound }\end{array}$ & & $\begin{array}{l}\text { Lower } \\
\text { bound }\end{array}$ & $\begin{array}{l}\text { Upper } \\
\text { bound }\end{array}$ \\
\hline Dutch Words RF & $2.25^{* * *}$ & 1.55 & 2.94 & $1.86^{* * *}$ & 1.20 & 2.52 & .25 & -.30 & .80 \\
\hline Pseudoword RF & $2.65^{* * *}$ & 1.91 & 3.40 & $1.79^{* * *}$ & 1.13 & 2.44 & $.75^{* * *}$ & .19 & 1.31 \\
\hline Loanword RF & $1.59^{* * *}$ & .97 & 2.22 & $1.25^{* * *}$ & .64 & 1.86 & .48 & -.07 & 1.03 \\
\hline English Word RF & $1.60^{* * *}$ & .98 & 2.23 & $1.20^{* * *}$ & .59 & 1.80 & .52 & -.03 & 1.07 \\
\hline Dutch Spelling & $1.77^{* * *}$ & 1.12 & 2.41 & $1.37^{* * *}$ & .76 & 1.99 & .50 & -.05 & 1.06 \\
\hline English Spelling & $2.42^{* * *}$ & 1.71 & 3.14 & $1.98^{* * *}$ & 1.30 & 2.65 & $.52^{*}$ & -.04 & 1.07 \\
\hline Dutch Vocabulary & $.70^{* * *}$ & .14 & 1.26 & $.61^{*}$ & .04 & 1.18 & .25 & -.29 & .80 \\
\hline English Vocabulary & $.71^{* * *}$ & .15 & 1.27 & $.57^{* *}$ & .01 & 1.14 & .20 & -.35 & .74 \\
\hline Spoonerisms - Accuracy & $1.70^{* * *}$ & 1.06 & 2.33 & $1.13^{* * *}$ & .53 & 1.72 & .66 & .10 & 1.21 \\
\hline Spoonerisms - RT (Log) & $-2.79^{* * *}$ & -3.55 & -2.02 & $-1.45^{* * *}$ & -2.07 & -.82 & $-1.18^{* * *}$ & -1.76 & -.59 \\
\hline RAN (Items / min) & $.94^{* * *}$ & .37 & 1.51 & $.70^{* *}$ & .13 & 1.28 & .20 & -.34 & .75 \\
\hline Digit Span - Forward & $1.20^{* * *}$ & .61 & 1.79 & .54 & -.03 & 1.10 & $.74^{* * *}$ & .18 & 1.31 \\
\hline Digit Span - Backward & $1.02^{* * *}$ & .44 & 1.59 & $.57^{* *}$ & .01 & 1.14 & .34 & -.21 & .89 \\
\hline
\end{tabular}

Note. The indicated statistical significance is based on pairwise comparisons using the Least Square Difference method in the MANOVA

${ }^{*} p<.01,{ }^{* *} p<.05,{ }^{* * *} p<.1$

LRnonDys $=$ Low Risk without Dyslexia, HRDys $=$ High Risk with Dyslexia, HRnonDys $=$ High Risk without Dyslexia, RF $=$ Reading Fluency, RT $=$ Reaction Time, RAN $=$ Rapid Automatized Naming, MANOVA = Multivariate Analysis of Variance. 